\title{
Two-color phase-controlled photoemission from a zero-dimensional nanostructure
}

\author{
Timo Paschen ${ }^{1, *}$, Michael Förster ${ }^{1}$, Michael Krüger ${ }^{1}$, Christoph Lemell ${ }^{2}$, Georg Wachter ${ }^{2}$, \\ Florian Libisch ${ }^{2}$, Thomas Madlener ${ }^{2}$, Joachim Burgdörfer ${ }^{2}$, and Peter Hommelhoff ${ }^{1,3}$ \\ ${ }^{1}$ Department of Physics, Friedrich-Alexander-Universität Erlangen-Nürnberg (FAU), \\ Staudtstrasse 1, 91058 Erlangen, Germany \\ ${ }^{2}$ Institute for Theoretical Physics, Vienna University of Technology, 1040 Vienna, Austria \\ ${ }^{3}$ Max Planck Institute for the Science of Light, Staudtstrasse 2, 91058 Erlangen, Germany
}

\begin{abstract}
We demonstrate that multi-photon photoemission including above-threshold multiphoton orders from a nanotip can be coherently controlled with the optical phase between two light fields. By focusing 74 fs drive pulses at $1560 \mathrm{~nm}$ and their second harmonic at $780 \mathrm{~nm}$ onto the tip and changing the optical phase between the two colors, we observe an emission current modulation of up to $97.5 \%$. Additionally, electron energy spectra reveal a homogeneous modulation of all multiphoton orders. Hence, the electron current can be strongly increased (by a factor of 3.7) or almost completely turned off due to interference between two different quantum channels in the material. We argue that the extremely high degree of coherence evidenced by this near-unity current modulation depth is due to the confinement of the local field enhancement at the nanotip. The nanorod effect allows to apply large DC fields, adding a further degree of freedom to investigate the modulation contrast of the photoemitted electron yield. We show that for an increasing DC electric field a non-cooperative distribution of electron emission leads to a decrease in modulation contrast.
\end{abstract}

The interaction of electrons with solid matter in intense laser fields has become a diverse research area within the physics community and opened the exciting field of attosecond nanoscale physics (for a comprehensive review, see [1,2]). For instance, the formation of hot plasmas due to the interaction of nano-particles and intense laser pulses or the observation of collective dynamics of bound charges in graphene-like structures has been made possible by combining attosecond timing precision and new advances in nanofabrication. Recently, attosecond control of photoemitted electrons from a solid-state system (i.e. nanotips) has been demonstrated as well [3]. Here we present further control of photoemitted electrons by using a synthesized two-color laser field for excitation $[4,5]$ and exploiting the optical phase (delay) between the two colors.

\footnotetext{
* Corresponding author: timo.paschen@,fau.de
} 

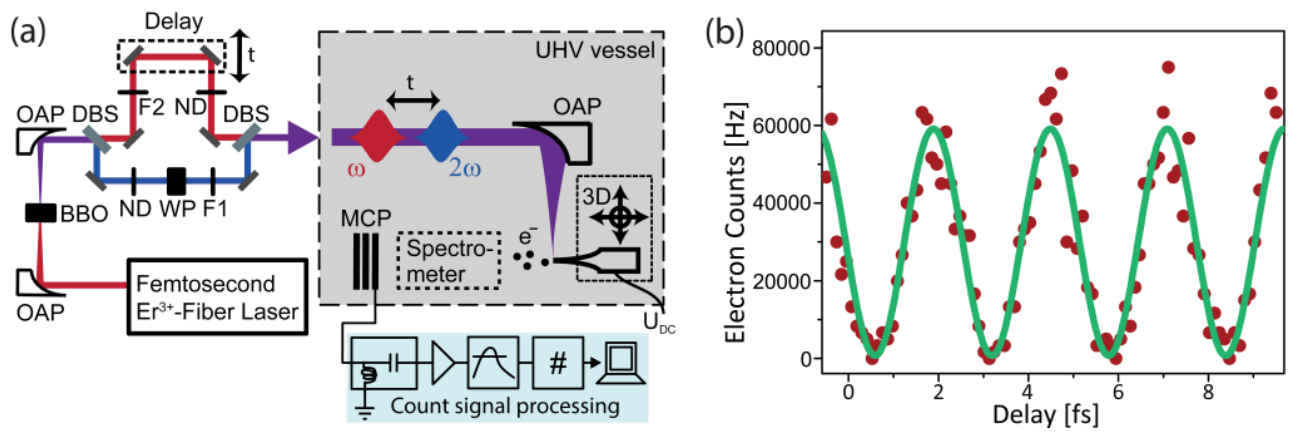

Fig. 1. (a) Experimental setup for two-color photoemission. Femtosecond laser pulses from an Erbium-doped fiber laser and its second harmonic are focused onto the nanotip to trigger two-color phase-controlled photoemission. (b) Electron count rate dependent on time delay $\tau$ or, equivalently optical two-color phase $\phi .97 .5 \%$ count rate modulation is achieved upon change of the optical phase between the two colors.

Ionization by two-color laser fields with well-defined relative phase allows one to tune and control electronic dynamics on the (sub-) femtosecond time scale. Two-color pulses have been used to investigate above-threshold ionization of atoms, controlled dissociative ionization, dichroism in ionization, and orientation of molecules. In our experiment, atomic-scale in situ control over the sample surface results in a well-defined nanoemitter that surpasses the limitations of focal averaging and inhomogeneous broadening. Electron emission induced by a strong fundamental pulse can be enhanced or suppressed with a contrast of up to $97.5 \%$ when superimposing a weak second harmonic pulse [4,5] (see Fig. 1(b)). This scheme allows for efficient coherent control of the nanotip photocurrent utilizing the metal-vacuum interface to suppress emission in one direction. Density functional theory simulations show a large surface density of states (doorway state) near $4 \hbar \omega$ suggesting that the strong modulation can be explained in terms of two nearly perfectly constructively or destructively interfering quantum pathways, each individually leading to electron emission

Two-color phase-resolved electron energy spectra reveal, while all multiphoton orders show an exponential drop in count rate towards higher electron energies, that each order exhibits the same dependence on the optical phase between the two colors (see Fig. 2(a)). Furthermore, the individual visibility extracted from each multiphoton order scales similar compared to the global visibility of the energy-integrated photoemission signal. From these findings, a simplified model involving two separate emission channels and resulting quantum pathway interference due to the coherent scheme of the two-color excitation can be developed (see setup in Fig. 1(a)).

The solid-state nature of the emitter allows for applying a DC electric field, which provides further control of the coherent electron emission. Strong DC fields of up to $-2 \mathrm{GV} / \mathrm{m}$ can be applied to the tip, changing the induced near-field landscape directly at the surface of the nanostructure. The visibility (see upper panel in Fig. 2(b)) of the coherently controlled emission is monotonically decreasing for increasing DC fields at the tip. This behavior is caused by the simultaneous increase of the non-cooperative electron emission contribution independent of the two-color phase (contribution $\mathrm{A}_{0}$ in the lower panel in Fig. 2(b)) which overshadows the modulation of the two-path interference. 
(a)

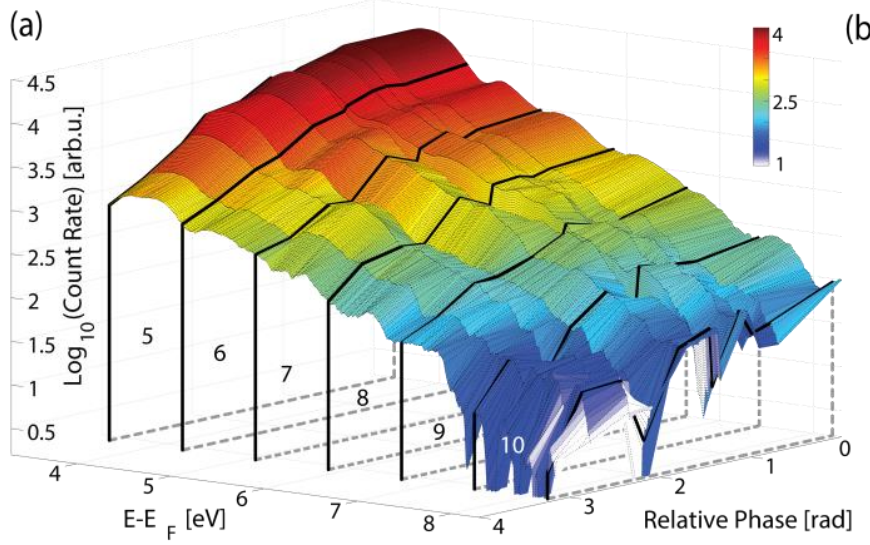

(b)

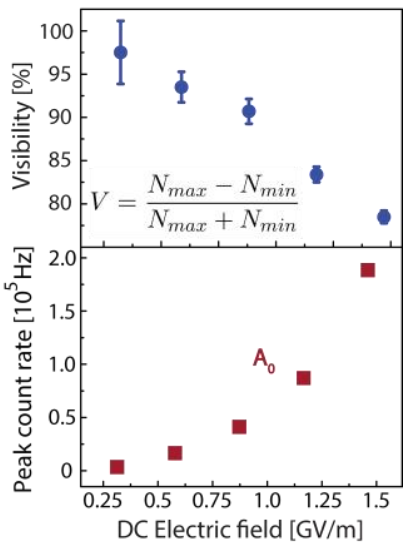

Fig. 2. (a) Two-color phase-dependent electron energy spectra. All above-threshold photon orders show a similar exponential drop in count rate towards higher energies for all two-color phases and the same phase-dependent modulation. (b) Modulation visibility $V$ of the photoemitted electrons and scaling of the DC part of the photoemission signal for different DC electric fields.

While the superb modulation contrast of electron emission from nanotips could be demonstrated for the perturbative regime (Keldysh $\gamma>>1$ ), experiments in the strong-field regime should provide insight in field-driven two-color coherent control. Here, it is expected that high-energy electrons in the cut-off region should be sensitive to the optical phase of the two colors [6]. Furthermore, due to the almost perfect control on the femtosecond duration and nanometer length scales the demonstrated photoemission system can be seen as a future building block of light wave electronics [7].

1. M. Ciappina, J. Pérez-Hernández, A. Landsman, W. Okell, S. Zherebtsov, B. Förg, J. Schötz, L. Seiffert, T. Fennel, T. Shaaran, T. Zimmermann, A. Chacón, R. Guichard, A. Zaïr, J. Tisch, J. Marangos, T. Witting, A. Braun, S. Maier, L. Roso, M. Krüger, P. Hommelhoff, M. Kling, F. Krausz, M. Lewenstein, Rep. Prog. Phys. 80, 054401 (2017).

2. M. Krüger, C. Lemell, G. Wachter, J. Burgdörfer, P. Hommelhoff, J. Phys. B: At. Mol. Opt. Phys. in press (2018).

3. M. Krüger, M. Schenk, P. Hommelhoff, Nature 475, 78 (2011).

4. M. Förster, T. Paschen, M. Krüger, C. Lemell, G. Wachter, F. Libisch, T. Madlener, J. Burgdörfer, P. Hommelhoff, Phys. Rev. Lett., 117, 217601 (2016).

5. T. Paschen, M. Förster, M. Krüger, C. Lemell, G. Wachter, F. Libisch, T. Madlener, J. Burgdörfer, P. Hommelhoff, J. Mod. Opt. 64, 10-11, 1054-1060 (2017).

6. L. Seiffert, T. Paschen, P. Hommelhoff, T. Fennel, J. Phys. B, 51, 134001 (2018).

7. F. Krausz, M. Stockman, Nat. Photonics, 8, 205 (2014). 